\title{
Lung cancer mortality in the French cohort of titanium dioxide workers: some etiological insights
}

Irina Guseva Canu ${ }^{1 *}$, Alan Gaillen-Guédy ${ }^{1}$, Pascal Wild ${ }^{1,2}$, Kurt Straif $^{3}$, and Danièle Luce ${ }^{4}$

${ }^{1}$ Center for primary care and public health (Unisanté), University of Lausanne, Switzerland

${ }^{2}$ National Institute of Safety Research (INRS), Nancy, France

${ }^{3}$ International Agency for Research on Cancer (IARC), Lyon, France

${ }^{4}$ Université de Rennes, Inserm, EHESP, Irset (Institut de recherche en santé, environnement et travail)

- UMRS 1085, Pointe-à-Pitre, France

Word count: 1463 words

Number of tables/figures: 1 table

Supplementary material: 3 tables

*Correspondance to:

Prof. GUSEVA CANU Irina

Département Santé-Travail-Environnement

Unisanté : Centre universitaire de médecine générale et de santé publique

Biopôle

Route de la Corniche, 2

CH-1066 Epalinges-Lausanne

Tel: +41213146224

E-mail: irinacanu@hotmail.com ; irina.guseva-canu@unisante.ch 


\section{Abstract}

Objectives: Titanium dioxide ( $\mathrm{TiO} 2)$ is widely used in construction, food, cosmetic and medical industry. The current evidence on $\mathrm{TiO} 2$ carcinogenicity in humans is considered inadequate. As French participants of the European cohort of $\mathrm{TiO} 2$ workers exhibited an increase in mortality from lung cancer, we aimed at investigating whether $\mathrm{TiO} 2$ exposure, co-exposures or smoking can explain this increase. Methods: We reanalyzed the data of 833 French male workers (follow-up period 1968-1997) and used multiple imputation to complete their smoking status. We considered respirable $\mathrm{TiO} 2$ dust as primary exposure of interest, estimated as continuous cumulative $(\mathrm{mg} / \mathrm{m} 3$-year) and annual average $(\mathrm{mg} / \mathrm{m} 3)$ concentrations and binary and 4-class categorical variables, with cut-off values of 0.3 and $2.4 \mathrm{mg} / \mathrm{m}^{3}$ (the German and American occupational exposure limits, respectively). For each exposure metric, we estimated hazard ratios and associated 95\%-confidence intervals (HR, 95\%-CI), using Cox regression models adjusted for calendar period, exposure duration and smoking.

Results: The fully adjusted model yielded a HR=3.7 (95\%CI=0.79-17.95) for TiO2-exposed workers versus unexposed and a $\mathrm{HR}=27.33(95 \% \mathrm{CI}=4.35-171.84)$ for those exposed to $>2.4 \mathrm{mg} / \mathrm{m} 3$ as annual average concentration. Employment duration was negatively related with lung cancer mortality, therefore cumulative exposure had a small effect on mortality $(\mathrm{HR}=1.03(95 \% \mathrm{CI}=0.99-1.08)$ per $\mathrm{mg} / \mathrm{m} 3$-year).

Conclusion: This study suggests a positive relationship between $\mathrm{TiO} 2$ exposure and lung cancer mortality in $\mathrm{TiO} 2$ workers, whatever the exposure variable used, despite a limited statistical power in some models. The results question the current evidence on $\mathrm{TiO} 2$ carcinogenicity in humans but need to be confirmed in other cohorts, using different statistical approaches.

Key words: titanium dioxide; respirable dust; inhalation; occupational exposure; healthy worker survivor effect 


\section{Key messages}

- What is already known about this subject?

Since 2006, $\mathrm{TiO} 2$ is classified as an IARC group 2B carcinogen (possibly carcinogenic to humans). In 2018, the European Chemicals Agency (ECHA) classified TiO2 under all forms as a category-2 carcinogen (suspected human carcinogen) by inhalation, given the inadequate evidence of its carcinogenicity in humans. Two large cohorts of American and European TiO2 workers reported statistically increased mortality from lung cancer, but failed to observe a doseresponse relationship with cumulative exposure to $\mathrm{TiO} 2$. Moreover, none of the analyses was adjusted for smoking.

\section{- What are the new findings?}

We re-analyzed the data of a French cohort of $\mathrm{TiO} 2$ workers, using four different $\mathrm{TiO} 2$ exposure variables and multiple imputations for missing data on tobacco smoking. We showed that relationship between the duration of exposure and lung cancer mortality is negative and masks the effect of $\mathrm{TiO} 2$ cumulative exposure, which remains of borderline significance, event after adjustment for smoking. Yet, relationship between annual average exposure and lung cancer mortality is consistent.

\section{- How might this impact on policy or clinical practice in the foreseeable future?}

If confirmed, these finding should trigger reconsidering the evidence on $\mathrm{TiO} 2$ carcinogenicity in humans, possibly upgrading the $\mathrm{TiO} 2$ classification. Moreover, it should trigger reconsidering the revision of the EU CLP Regulation for Classification, labeling and packaging of chemicals and ascribing $\mathrm{TiO} 2$ on the Annex IV of this regulation. 


\section{Introduction}

Titanium dioxide (TiO2) is an odorless white pigment and opacifying agent widely used since $1920 .{ }^{1}$ $\mathrm{TiO} 2$ is classified as possibly carcinogenic to humans by IARC and, since 2018 , as suspected human carcinogen by inhalation by the European Chemical Agency. ${ }^{1}$ Statistically increased lung cancer mortality was reported in two cohorts of American and European TiO2 workers. ${ }^{23}$

In the American cohort, the association between $\mathrm{TiO} 2$ exposure and cause-specific mortality was first investigated by Poisson regression, ${ }^{3}$ and, in accordance with the European analysis, ${ }^{2}$ using Cox regression. ${ }^{4}$ The authors reported a statistically significant increase of the hazard ratio (HR) for all cancer mortality but no results for lung cancer mortality. ${ }^{4}$ In the European study pooling cohorts from six countries, ${ }^{2}$ solely French participants experienced borderline significant increases in both all cancer $(\mathrm{SMR}=1.21(95 \% \mathrm{CI}=1.01-1.44))$ and lung cancer mortality $(\mathrm{SMR}=1.42(95 \% \mathrm{CI}=0.99-1.96))$, but the dose-response results were reported solely for the pooled cohort and were not adjusted for smoking. ${ }^{2}$

This study aimed at investigating the association between $\mathrm{TiO} 2$ exposure and lung cancer mortality among French participants of the European cohort, accounting for smoking and other potential confounders.

\section{Methods}

\subsection{Study sample, follow up and main outcome}

We re-analyzed the French data used in the dose-response analyses of the European cohort study. ${ }^{2}$ The included workers were men employed before 1990, for at least one year in a French TiO2 factory and had sufficiently complete data on demographic characteristics and on occupational history. Workers employed only in non-production jobs, and those for whom $25 \%$ of occupational history or more than 5 years were missing were excluded. Vital status and causes of death were assessed by the National Natural Persons Identification Index and the National Cause of Death Registry, respectively. The latter contains anonymized records of all deaths in France since 1968 and their causes. Death records were matched to cohort members by date of birth, gender, and date and place of death. The causes of death were coded according to the 9th revision of the International Classification of Diseases (ICD-9). As primary outcome we considered lung cancer deaths specified as underlying cause on the death certificate (ICD-9 code 162) that occurred over the period 1968-1997. 


\subsection{Exposure assessment}

We re-used the exposure data collected in the context of the European study. ${ }^{5}$ An occupational hygienist visited each factory, including the French one and collected information on exposure circumstances and files with relevant measurement data. All data were entered into a computer database and converted to a common exposure metric using appropriate conversion factors. ${ }^{5}$ Exposure levels were then estimated for respirable $\mathrm{TiO} 2$ dust, sulphuric acid mist, hydrochloric acid, asbestos and welding fumes at the level of occupational titles for discrete time-periods throughout the factory's operational history. Estimated exposure levels were then linked to the occupational history of each workers to provide estimates of cumulative occupational exposure.

\subsection{Tobacco smoking}

Initially, the factory nurse checked the medical records of workers present in the last five years and abstracted information on smoking status whenever available. ${ }^{2}$ To expand the usage of available data, we used multiple imputation method for the missing smoking data. We created a multinomial logistic regression imputation model with a possible outcome of 1 for non-smoker, 2 for ex-smoker and 3 for smoker using the following variables: the year of birth, the years of employment, the age at the start of employment, the age at the end of follow-up, the total duration of employment, the different types of work performed as well as their respective durations. Using this model and the existing data on smoking, we run 1000 smoking status imputations for workers with missing data. The different Cox models (see below), adjusted on the smoking categories were run for each of the 1000 imputations. The estimates were then combined using Royston's method. ${ }^{6}$

\subsection{Statistical analysis}

We considered respirable $\mathrm{TiO} 2$ dust as a binary variable (yes/no), then by cumulative exposure lagged by 10 years (mg/m3-year) and by duration of employment in years; we computed the ratio of these two variables to calculate the annual average exposure $(\mathrm{mg} / \mathrm{m} 3)$. We also considered the annual average exposure as a 4-class categorical variables (unexposed, ]0-0.3], ]0.3-2.4], and >2.4 mg/m $\mathrm{m}^{3}$ ), with cut-off values determined by the currently recommended occupational exposure limits (OEL) in Germany and the USA. ${ }^{78}$ All other exposures were treated as co-exposures. We implemented several Cox regression models (with age as the main time variable). Models were first adjusted for calendar period (before 1975 then by 5 -year periods until 1997), then for employment duration and if relevant, for the co-exposure. 
To control for potential confounding by smoking, we first ran all models without adjustment on smoking, second with the adjustment using the multiple imputed smoking categories. Sensitivity analysis consisted of testing 0,5 , and 15-year lag on the cumulative $\mathrm{TiO} 2$ exposure to account for a latency period. Data were analyzed using Stata version 15.

\section{Results}

The cohort comprised 833 male workers (17’390 person-years) among whom 75\% were exposed to TiO2 (Supplementary material tables S1 and S2). Other dusts and sulfuric acid were the most common co-exposures. However, none of the co-exposure was associated with $\mathrm{TiO} 2$ exposure and the outcome (results not shown) and therefore not included in multivariate models. Smoking status was known for $61 \%$ of workers, $5 \%$ of whom smoked (Table S1). At the end of follow-up, $13 \%$ of workers were deceased, with 16 lung cancer deaths in total. Compared to unexposed, TiO2-exposed workers exhibited an approximately 4-fold higher risk of lung cancer mortality, though statistically non-significant (Table 1). The analysis according to the annual average exposure showed a significant increase in lung cancer mortality per $\mathrm{mg} / \mathrm{m} 3$ of respirable $\mathrm{TiO} 2$ dust exposure. The adjustment for exposure duration decreased the HRs, while adjustment for smoking slightly increased them. The fully adjusted model resulted in a $\mathrm{HR}=2.07$ (95\%CI=1.34-3.20), i.e, an approximately twofold increased risk of lung cancer mortality per increment of one $\mathrm{mg} / \mathrm{m} 3$ of respirable $\mathrm{TiO} 2$ dust exposure as annual average concentration. In contrast, the exposure duration was negatively related to the outcome (data not shown). Therefore, the analysis according to cumulative exposure to respirable $\mathrm{TiO} 2$ dust resulted only in a small increase of lung cancer mortality ( 2 to $4 \%$ per $\mathrm{mg} / \mathrm{m} 3$-year of respirable $\mathrm{TiO} 2$ dust exposure, depending on the lag duration (Table 1), though of borderline statistical significance. Smoking appeared not to confound any of these associations.

\section{Discussion}

This study is based on a small cohort of French $\mathrm{TiO} 2$ exposed workers with available $\mathrm{TiO} 2$ exposure data, and only a few lung cancer deaths, especially among non-exposed workers. This calls for a careful result interpretation. This calls for a careful result interpretation. However, a positive exposure-response relationship based on continuous annual average exposure found in this study, seems quite precise and robust to adjustment for confounders. This result is consistent with results based on all other $\mathrm{TiO}_{2}$ 
exposure variables used, despite imprecision of some HRs, and questions the previously suggested absence of a relationship between $\mathrm{TiO}_{2}$ exposure and lung cancer in humans.

Adjustment for smoking had no effect on these results. However, the prevalence of smoking in our cohort seems unusually low. Insufficient quality of the smoking data due to potential underreporting of smoking by workers during the 90ths is likely to explain this, although we cannot verify this assumption. Previous studies focused on the cumulative exposure as continuous or categorical variable, and observed no statistically significant trend with lung cancer incidence and/or mortality. However, here, we observed that the exposure duration is negatively associated with the outcome. As shown in Table S3, the most heavily exposed workers left their employment much earlier than their less exposed colleagues did, suggesting the presence of a healthy worker survivor effect (HWSE). HWSE impedes dose-response analysis with cumulative exposure variables using Cox regression and therefore undermines the relevance of the results ${ }^{9}$. Therefore, the HRs should be interpreted with caution.

Today, only experimental studies documented the carcinogenic potential of $\mathrm{TiO} 2 .{ }^{10}$ The latter was systematically observed in highly exposed rats, as result of "particle lung overload" that exceeds the particle clearance ability of alveolar macrophages. ${ }^{11}$ Given the anatomic differences between rats and humans, and lack of evidence from previous epidemiological studies, the relevance of the carcinogenic effect of $\mathrm{TiO} 2$ at high doses for humans remains controversial. Recent experimental studies elucidated TiO2 inflammogenic potential and capacity to elicit pulmonary toxicity with contribution from neutrophils and macrophages independent of the overload phenomenon. ${ }^{11}$ A secondary genotoxic mechanism of $\mathrm{TiO} 2$, through oxidative stress, was recently evidenced for $\mathrm{TiO} 2$ nanoparticles. ${ }^{12}$ However, the nuclear transfer of $\mathrm{TiO} 2$ nanoparticles suggests the likelihood of primary genotoxicity, through direct interaction with DNA. ${ }^{12}$

Although, no physiochemical characterization of $\mathrm{TiO} 2$ was possible in this study, we assume the workers have handled essentially pigment-grade microparticulated $\mathrm{TiO} 2$. Our results should be confirmed in other national cohorts of $\mathrm{TiO} 2$ workers, using different statistical approaches. In particular, G-methods, enabling correction for the HWSE, should be implemented as a next analytical step for the European cohort of $\mathrm{TiO} 2$ workers along with a better physical-chemical characterization of $\mathrm{TiO} 2$ in future studies. 
Compliance with Ethical Standards: The use of the individual data was approved by the French Data Protection Authority (CNIL), Authorization No 999250.

Funding: This work was supported by the University of Lausanne.

Conflict of Interest: The authors declare no conflicts of interest.

Acknowledgment: The authors thank S. Charles and C. Michel from the French Agency for Food, Environmental and Occupational Health \& Safety (ANSES) for their help in accessing the data. 


\section{References}

1. Guseva Canu I, Fraize-Frontier S, Michel C, et al. Weight of epidemiological evidence for titanium dioxide risk assessment: current state and further needs. J Expo Sci Environ Epidemiol 2019 doi: 10.1038/s41370-019-0161-2 [published Online First: 2019/08/20]

2. Boffetta P, Soutar A, Cherrie JW, et al. Mortality among workers employed in the titanium dioxide production industry in Europe. Cancer Causes Control 2004;15(7):697-706. doi:

\subsection{3/B:CACO.0000036188.23970.22}

3. Ellis ED, Watkins JP, Tankersley WG, et al. Occupational exposure and mortality among workers at three titanium dioxide plants. Am J Ind Med 2013;56(3):282-91. doi: 10.1002/ajim.22137

4. Golden A, Ellis ED, Le H. Comparison of risk estimates from cox proportional hazards and poisson modeling for association of occupational titanium dioxide exposure and selected causes of death. Occup Environ Med 2017;74(S1):A49.

5. Cherrie JW, Schneider T, Spankie S, et al. A new method for structured, subjective assessments of past concentrations. Occup Hyg 1996;3:75-83.

6. Royston P. Multiple imputation of missing values: Further update of ice, with an emphasis on categorical variables. Stata Journal 2009;9:466-77.

7. MAK. Titanium dioxide (respirable fraction) [MAK Value Documentation, 2009]. The MAKCollection for Occupational Health and Safety:1-66.

8. US-NIOSH. Current Intelligence Bulletin 63. Occupational Exposure to Titanium Dioxide2011.

9. Picciotto S, Hertz-Picciotto I. Commentary: healthy worker survivor bias: a still-evolving concept. Epidemiology 2015;26(2):213-5. doi: 10.1097/ede.0000000000000233 [published Online First: 2015/02/03]

10. Johnston HJ, Hutchison GR, Christensen FM, et al. Identification of the mechanisms that drive the toxicity of $\mathrm{TiO}(2)$ particulates: the contribution of physicochemical characteristics. Particle and fibre toxicology 2009;6:33-33. doi: 10.1186/1743-8977-6-33

11. Li W, Pauluhn J. Re-defining kinetic lung overload: Time for new paradigms. Toxicology letters 2018;295:212-19. doi: 10.1016/j.toxlet.2018.06.1222 [published Online First: 2018/07/04] 
12. Charles S, Jomini S, Fessard V, et al. Assessment of the in vitro genotoxicity of TiO2 nanoparticles in a regulatory context. Nanotoxicology 2018;12(4):357-74. doi:

10.1080/17435390.2018.1451567 [published Online First: 2018/03/20] 
Table 1. Association between different $\mathrm{TiO}_{2}$ exposure metrics and lung cancer mortality in French $\mathrm{TiO}_{2}$ workers (1968-1997)

\begin{tabular}{|c|c|c|c|c|c|c|c|}
\hline TiO2 exposure metric & $\begin{array}{c}\text { Observed } \\
\text { lung } \\
\text { cancer } \\
\text { deaths } \\
\end{array}$ & & Model 1* & & Model 2* & & Model 3* \\
\hline Binary exposed vs non-exposed & 14 & 3.75 & [0.79-17.9] & 4.34 & {$[0.85-22.15]$} & 3.77 & [0.79-17.95] \\
\hline \multicolumn{8}{|l|}{ Categorical annual average exposure vs non-exposed } \\
\hline ]0-0.3] mg/m 3 & 7 & 4.04 & {$[0.79-20.63]$} & 5.94 & {$[1.07-32.99]$} & 4.15 & {$[0.81-21.21]$} \\
\hline$[0.3-2.4] \mathrm{mg} / \mathrm{m}^{3}$ & 3 & 1.68 & {$[0.26-10.93]$} & 1.64 & {$[0.24-11.11]$} & 1.64 & {$[0.25-10.67]$} \\
\hline$>2.4 \mathrm{mg} / \mathrm{m}^{3}$ & 4 & 28.28 & {$[4.57-175.15]$} & 12.97 & {$[1.86-90.74]$} & 27.33 & {$[4.35-171.84]$} \\
\hline Continuous annual average exposure $(\mathrm{mg} / \mathrm{m} 3)$ & 16 & 2.10 & {$[1.37-3.22]$} & 1.70 & {$[1.03-2.79]$} & 2.07 & {$[1.34-3.20]$} \\
\hline Continuous cumulative exposure ( $\mathrm{mg} / \mathrm{m} 3$-year), 0 lag & 16 & 1.02 & {$[0.97-1.06]$} & - & - & 1.02 & {$[0.97-1.06]^{* * *}$} \\
\hline 5-year lag & $9 * *$ & 1.02 & {$[0.98-1.07]$} & - & - & 1.02 & {$[0.98-1.07]^{* * *}$} \\
\hline 10-year lag & $5 * *$ & 1.03 & {$[0.99-1.08]$} & - & - & 1.03 & {$[0.98-1.08]^{* * *}$} \\
\hline 15-year lag & $1 * *$ & 1.04 & {$[0.98-1.11]$} & - & - & 1.04 & {$[0.98-1.11]^{* * *}$} \\
\hline
\end{tabular}

